\title{
Slow oscillations in blood pressure via a nonlinear feedback model
}

\author{
JOHN V. RINGWOOD ${ }^{1}$ AND SIMON C. MALPAS ${ }^{2}$ \\ ${ }^{1}$ Department of Electronic Engineering, National University of Ireland, Maynooth, \\ County Kildare, Ireland; and ${ }^{2}$ Circulatory Control Laboratory, \\ Department of Physiology, University of Auckland, Auckland, New Zealand
}

Received 14 January 2000, accepted in final form 1 November 2000

\begin{abstract}
Ringwood, John V., and Simon C. Malpas. Slow oscillations in blood pressure via a nonlinear feedback model. Am J Physiol Regulatory Integrative Comp Physiol 280: R1105-R1115, 2001.-Blood pressure is well established to contain a potential oscillation between 0.1 and $0.4 \mathrm{~Hz}$, which is proposed to reflect resonant feedback in the baroreflex loop. A linear feedback model, comprising delay and lag terms for the vasculature, and a linear proportional derivative controller have been proposed to account for the $0.4-\mathrm{Hz}$ oscillation in blood pressure in rats. However, although this model can produce oscillations at the required frequency, some strict relationships between the controller and vasculature parameters must be true for the oscillations to be stable. We developed a nonlinear model, containing an amplitude-limiting nonlinearity that allows for similar oscillations under a very mild set of assumptions. Models constructed from arterial pressure and sympathetic nerve activity recordings obtained from conscious rabbits under resting conditions suggest that the nonlinearity in the feedback loop is not contained within the vasculature, but rather is confined to the central nervous system. The advantage of the model is that it provides for sustained stable oscillations under a wide variety of situations even where gain at various points along the feedback loop may be altered, a situation that is not possible with a linear feedback model. Our model shows how variations in some of the nonlinearity characteristics can account for growth or decay in the oscillations and situations where the oscillations can disappear altogether. Such variations are shown to accord well with observed experimental data. Additionally, using a nonlinear feedback model, it is straightforward to show that the variation in frequency of the oscillations in blood pressure in rats $(0.4$ $\mathrm{Hz})$, rabbits $(0.3 \mathrm{~Hz})$, and humans $(0.1 \mathrm{~Hz})$ is primarily due to scaling effects of conduction times between species.
\end{abstract}

sympathetic nervous system; baroreflex; stability; describing function; artificial neural network

IT IS WELL ESTABLISHED that blood pressure in humans can contain a distinct oscillation at $0.1 \mathrm{~Hz}$, often referred to as the Mayer wave $(26,38)$. Experiments in a variety of animal models have shown that this oscillation is due to the action of the sympathetic nervous system on the vasculature. Although the oscillation in

\footnotetext{
Address for reprint requests and other correspondence: S. C. Malpas, Circulatory Control Laboratory, Dept. of Physiology, Univ. of Auckland Medical School, Private Bag 92109, Auckland, New Zealand (E-mail: s.malpas@auckland.ac.nz).
}

blood pressure is shifted to $0.4 \mathrm{~Hz}$ in the rat (7) and to $0.3 \mathrm{~Hz}$ in the rabbit (22), changes in the strength of this oscillation have been proposed to reflect changes in the mean level of sympathetic nerve activity (SNA) and/or baroreflex gain (6), raising the possibility that measurement of the strength of this oscillation may be used as a diagnostic measure of neural control of the cardiovascular system in humans $(1,10,26)$.

Current evidence favors the concept of feedback in the baroreflex loop as the origin for the $0.1-\mathrm{Hz}$ oscillation in blood pressure $(5,6,13,25,41)$. In this model, a change in blood pressure is sensed by the arterial baroreceptors altering the afferent signal to the central nervous system (CNS) and subsequently the mean SNA level, which, in turn, alters vascular tone in the target organ. In the feedback representation, changes in blood pressure at a certain frequency undergo a phase shift of -180 degrees, which, combined with the negative feedback sign $(-1)$, results in positive feedback, which sustains the oscillation at that frequency.

Burgess et al. (8) proposed a linear feedback model to account for this oscillation, adopting a particular form of linear controller to represent the neural controlling mechanism, employing both mean arterial pressure (MAP) and rate-of-change of MAP (proportional-derivative, or PD) to determine the SNA signal. However, this structure requires a very strict relationship between the vasculature and controller parameters to exist to maintain sustained (and stable) oscillations. Thus stimuli that alter gain along the feedback loop (e.g., altered baroreflex gain) would predispose the oscillation toward either instability (gain increase) or asymptotic stability (gain decrease) and it would increase without bound or cease altogether. For a stable oscillation to be maintained during such changes, a linear model implies continuous adaptation. Such a possibility would suggest that the oscillation is deliberate and has a useful function, as yet unknown, but is not simply a by-product of time delays in the baroreflex loop.

In the present study, we explore the hypothesis that a nonlinear feedback model is better able to explain the

\footnotetext{
The costs of publication of this article were defrayed in part by the payment of page charges. The article must therefore be hereby marked "advertisement" in accordance with 18 U.S.C. Section 1734 solely to indicate this fact.
} 
low-frequency oscillation, without the requirement for strict parametric relationships. This nonlinear model is also able to explain the frequency variability of the oscillation across different species and goes some way toward explaining the amplitude variation and presence/absence of the oscillation under certain physiological conditions. In addition, we test whether the nonlinearity is confined to the CNS or also extends to the vasculature response to SNA by constructing models from blood pressure and SNA data collected in conscious rabbits.

$\begin{aligned} \text { Glossary } & \\ \text { SNA } & \text { Sympathetic nerve activity } \\ \text { MAP } & \text { Mean arterial pressure } \\ \text { CNS } & \text { Central nervous system } \\ g() & \text { Nonlinearity (static) in central nervous } \\ & \text { system } \\ \tau_{e_{1}} & \text { Preganglionic (efferent) delay } \\ \tau_{e_{2}} & \text { Postganglionic (efferent) delay } \\ v() & \text { Nonlinearity (static) in vasculature } \\ G_{v}(s) & \text { Linear vasculature dynamics } \\ G_{b}(s) & \text { Linear baroreceptor dynamics } \\ \tau_{a} & \text { Afferent delay } \\ \tau_{e} & \text { Total efferent delay } \\ K(s) & \text { Burgess' CNS controller } \\ G(s) & \text { Burgess' vasculature dynamics } \\ H(s) & \text { Burgess' feedback dynamics (afferent de- } \\ & \text { lay) } \\ \tau_{v} & \text { Lag in vasculature dynamics } \\ k_{p} & \text { Proportional control gain } \\ k_{d} & \text { Derivative control gain } \\ k_{d}^{*} & \text { Derivative control gain as a multiplier on } \\ N(a) & \text { Combination nonlinearity in baroreflex } \\ & \text { loop } \\ g(x) & \text { Generalized sigmoidal characteristic } \\ \left(x^{*}, y^{*}\right) & \text { Center of symmetry of sigmoid character- } \\ r^{*} & \text { istic } \\ \alpha, \beta & \text { Vertical range of sigmoid characteristic } \\ w_{o} & \text { Frequency of oscillation, rads/s } \\ G(z) & \text { Discrete-time vasculature dynamics } \\ n & \text { Degree of denominator of } G(z)\end{aligned}$

$\begin{aligned} m & \text { Degree of numerator of } G(z) \\ d & \text { Number of steps delay in } G(z) \\ a_{i} & \text { Denominator coefficients in } G(z) \\ \mathrm{ARX} & \text { AutoRegressive with eXogenous input } \\ & \text { model } \\ \mathrm{MSE}_{\mathrm{MAP}} & \begin{array}{l}\text { Mean squared error in MAP model pre- } \\ \text { diction }\end{array} \\ \mathrm{MAP}_{a} & \text { Actual MAP value } \\ \mathrm{MAP}_{m} & \text { Modeled MAP value } \\ N & \text { Number of data points }\end{aligned}$

\section{METHODS}

For comparative purposes, model parameters previously published by Burgess et al. (8) for rats were used to compare the efficacy of a linear vs. nonlinear model in the synthesis of an oscillation at $0.4 \mathrm{~Hz}$ in rats.

Subsequently, to validate the model further and explore the location of the nonlinearity, resting levels of SNA and MAP were recorded in conscious rabbits (weight 2.5-3.0, mean $2.6 \mathrm{~kg}, 5$ rabbits in total) for a 35 -min period. Animals underwent surgery, at least 7 days before the recording, to implant a recording electrode around the left renal sympathetic nerve as previously described (28). Experiments were previously approved by the University of Auckland Animal Ethics Committee. Arterial pressure was measured from a catheter inserted in a central ear artery. SNA was amplified, filtered between 50 and $5,000 \mathrm{~Hz}$, full-wave rectified, and integrated using a low-pass filter with a time constant of 20 ms. This integrated SNA signal and arterial pressure were continuously recorded throughout the experiment and were sampled at $500 \mathrm{~Hz}$ using an analog-to-digital data-acquisition card (National Instruments). Calibrated signals were displayed on a computer screen and saved to disk using a program written in the LabVIEW graphical programming language (National Instruments). For model development, data were subsequently filtered using a seventh-order Butterworth filter at $1 \mathrm{~Hz}$ and resampled at $2 \mathrm{~Hz}$.

\section{RESULTS}

\section{A Mathematical Vasculature Model}

A block diagram for the vasculature and the CNS is shown in Fig. 1.

The pure time delays $\tau_{a}$ and $\tau_{e}=\tau_{e_{1}}+\tau_{e_{2}}$ are due to conduction time along the efferent and afferent nerves and neurotransmission.
Fig. 1. Block diagram of the central nervous system and vasculature that give rise to the components of the feedback model (see Glossary for definitions).

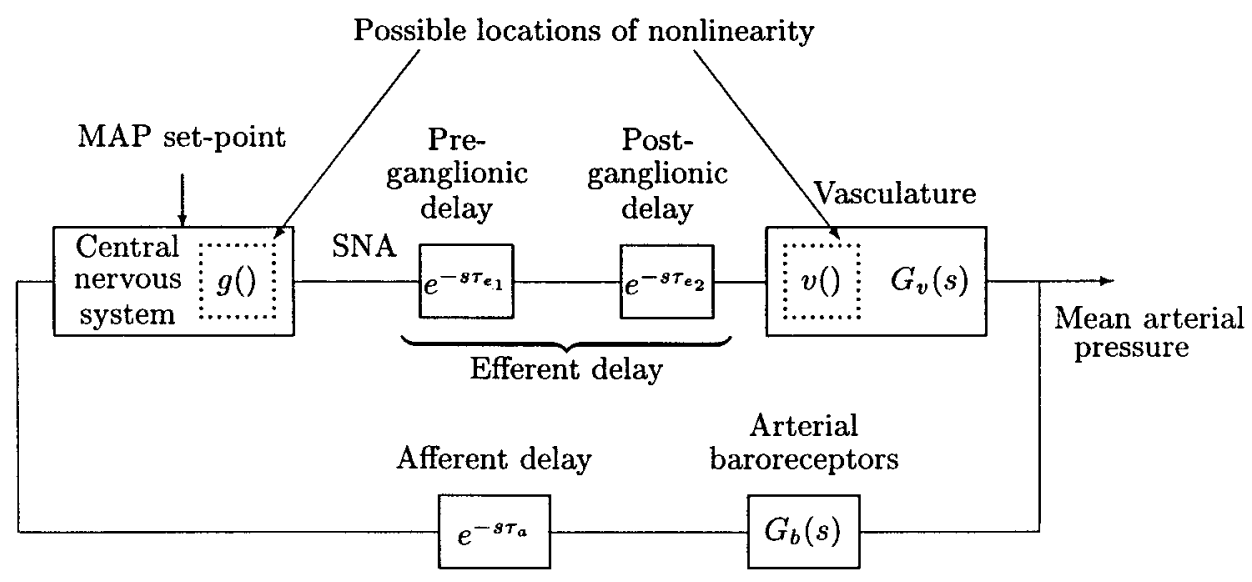




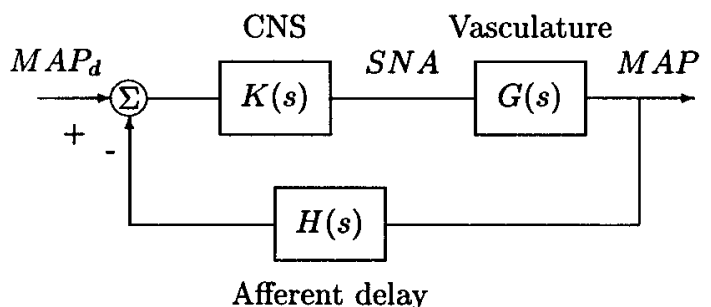

Fig. 2. Components of the linear feedback system developed by Burgess et al. (8).

\section{A Linear Feedback Model}

In a study by Burgess et al. (8) on rats, a model of the form shown in Fig. 2 is assumed, which includes both the vasculature and the CNS, where

$$
G(s)=\frac{e^{-s \tau_{e}}}{1+s \tau_{v}}, \quad H(s)=e^{-s \tau_{a}}, \quad K(s)=k_{p}+k_{d} \mathbf{S}
$$

The equivalence to the generalized diagram of Fig. 1 is obtained by the identities

$$
g()=K(s), v()=1, \quad G(s)=G_{v}(s) e^{-s \tau_{e}}, \quad G_{b}(s)=1
$$

Note that the controller $K(s)$ is $\mathrm{PD}(12)$ in form and $k_{\mathrm{d}}$ is expressed as a function of $\tau_{v}$ as

$$
k_{d}=k_{d}^{*} \tau_{v}
$$

The rational for the introduction of a $\mathrm{PD}$ controller is the observation that the baroreceptors respond both to magnitude and rate of change of MAP. It appears that the determination of $k_{p}$ and $k_{d}$ is performed based on the best fit to the observed data. In the study on seven different animals, Burgess et al. specify the parameters of $G(s)$ and determine the parameters of $K(s)$ to produce sustained oscillations at $0.4 \mathrm{~Hz}$ in MAP. The afferent delay in $H(s)$ is assumed constant at $0.2 \mathrm{~s}$. As

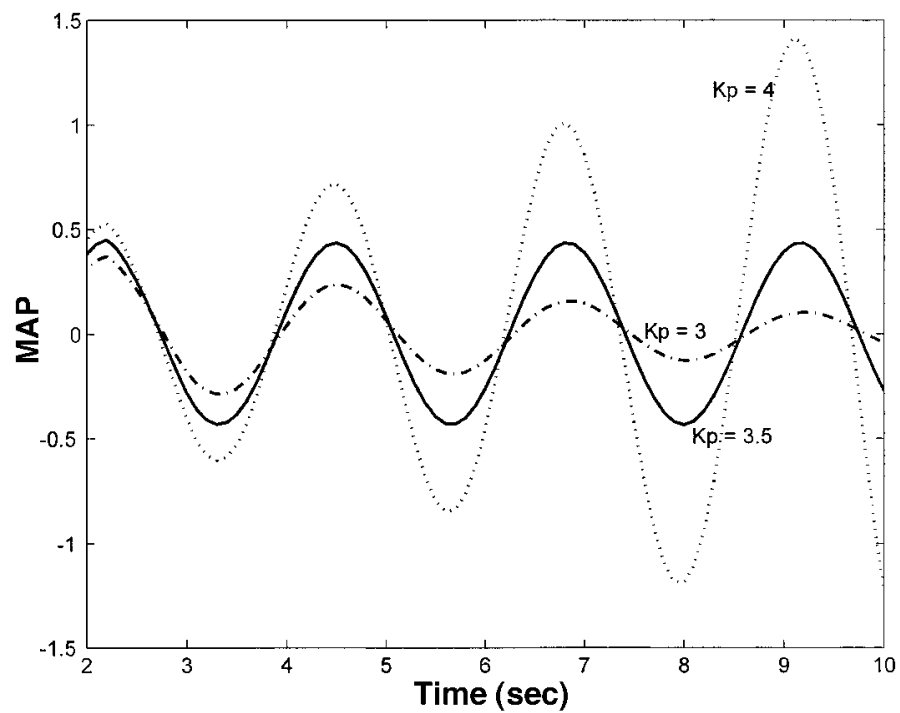

Fig. 3. Variations in mean arterial pressure (MAP) $(\mathrm{mmHg})$ around the mean level derived using a proportional-derivative controller, which indicates that the oscillation is difficult to sustain without the oscillation either growing $\left(K_{p}=4\right.$, dotted line $)$ or decaying $\left(K_{p}=3\right.$,

\begin{tabular}{|c|c|c|c|c|}
\hline $\begin{array}{c}\text { Efferent } \\
\text { Delay } \\
\left(\tau_{e}\right), \mathrm{s}\end{array}$ & $\begin{array}{c}\text { Vasculature } \\
\operatorname{Lag}\left(\tau_{v}\right), \mathrm{s}\end{array}$ & $\begin{array}{c}\text { Afferent } \\
\text { Delay } \\
\left(\tau_{a}\right), \mathrm{s}\end{array}$ & $\begin{array}{l}\text { Proportional } \\
\text { Gain }(k \mathrm{p}), \\
\frac{\mu \mathrm{V}}{\mathrm{mmHg}}\end{array}$ & 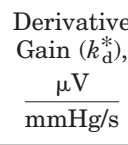 \\
\hline 0.6 & 1.3 & 0.2 & 3.5 & 0.37 \\
\hline
\end{tabular}
dashed line).
Table 1. Parameters for rat $E$

Taken from Burgess et al. (8).

an example, Fig. 3 shows the response in MAP (solid line) for the case shown in Table 1.

However, using a linear feedback model, these oscillations are difficult to sustain, without the oscillation amplitude either growing or decaying. This is illustrated in Fig. 3 for small variations in $k_{p}$. Increasing $k_{p}$ gives an unstable response, while decreasing $k_{p}$ results in asymptotic stability and the oscillation dies out. Burgess et al. determined the required condition for marginal stability as

$$
\sqrt{\frac{k_{p}^{2}-1}{1-k_{d}^{2}}} \frac{\left(\tau_{v}+\tau_{a}\right)}{\tau_{e}}-\cos ^{-1}\left(-\frac{1+k_{p} k_{d}}{k_{p}+k_{d}}\right)=0
$$

A corollary of this requirement is that any variation in the parameters of the vasculature, due to hormonal effects, etc., must be accompanied by a corresponding adjustment in the other (e.g., CNS) parameters, to sustain the marginal stability condition (4). This implies that the feedback loop is continuously self-adaptive.

\section{A Nonlinear Feedback Model}

Our proposal of a nonlinear feedback model is motivated by the following: 1) sustained oscillations are easily supported by a nonlinear feedback model; 2) the oscillations are stable over a wide variety of parameter variations, i.e., physiological conditions; and 3) a number of researchers $(4,37)$ demonstrated a sigmoidal nonlinearity in the baroreflex, particularly in the relationship between SNA and MAP, i.e., in the CNS.

Our proposed nonlinear feedback model is shown in Fig. 4, which is similar to that in Fig. 2, with the following exceptions: 1) the PD controller has been replaced with a proportional controller, and 2) there is an amplitude-limiting sigmoidal nonlinearity in the forward path. This could belong to either the neural controller or the vasculature itself.

Note that a nonlinear oscillation model does not preclude the use of a PD (or other) form of "controller" representation in the CNS. The choice of a gain is used purely for simplicity. Assuming a globally uniform relationship between blood pressure and SNA, ${ }^{1}$ a sigmoidal characteristic is proposed in Fig. 5 where

$$
y=g(x)=\frac{r^{*}}{1+\alpha e^{-\beta\left(x-x^{*}\right)}}-\frac{r^{*}}{1+\alpha e^{\beta\left(x-x^{*}\right)}}+y^{*}
$$

Note that this representation is separate from the negative feedback (-1) effect, accounting for the "for-

\footnotetext{
${ }^{1}$ This is clearly not the case in all situations, see Limitations.
} 


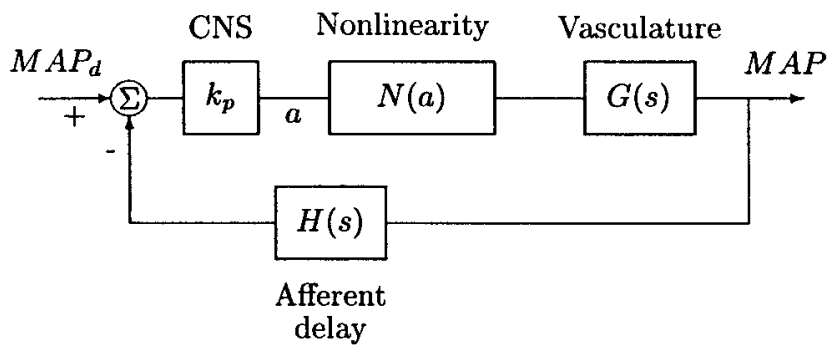

Fig. 4. Components of the proposed nonlinear feedback loop (see Glossary for definitions).

ward" S shape, as opposed to the more familiar reverse $\mathrm{S}$ seen more commonly in the baroreflex literature. The parameters $r^{*}, \alpha, \beta, x^{*}$, and $y^{*}$ specify the shape and position of the characteristic. It is known $(4,37)$ that a sigmoidlike characteristic represents the steady-state relationship between MAP and SNA and that the parameters describing the function vary with physiological condition. Figure 6 shows some of the possibilities, with the vasculature parameters specified in A Linear Feedback Model. Note also that the sigmoid as shown includes the effect of negative feedback (as shown in Fig. 4), accounting for the alternative orientation of the sigmoid curve compared to that normally presented for the arterial baroreflex.

The MAP responses in Fig. 6 correspond to the sigmoid parameters shown in Table 2, with $\alpha$ set equal to unity.

\section{Stability Analysis}

A stability analysis, following the Nyquist stability analysis of Burgess et al. (8), is also possible in the nonlinear case. However, the extension of linear frequency-domain techniques to the nonlinear case requires the introduction of the describing function (2), which attempts to represent the nonlinear element as a nonlinear gain. The resultant describing function, along with the $G H(j \omega)$ curve pertaining to the linear elements in Fig. 4, is plotted in Fig. 7.

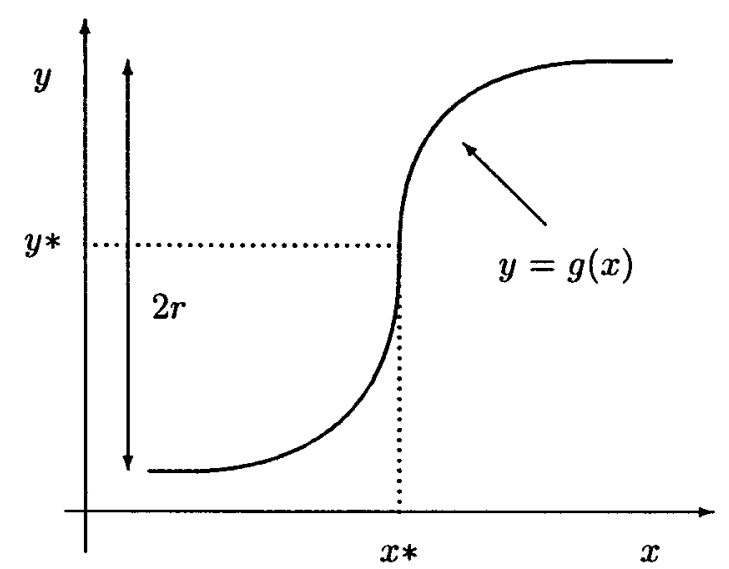

Fig. 5. Amplitude-limiting sigmoid characteristic between $\operatorname{MAP}\left(x^{*}\right)$ and sympathetic nerve activity (SNA; $\left.y^{*}\right)$. Note that this assumes a globally uniform relationship between blood pressure and SNA, which is not the case in all situations (see Limitations).

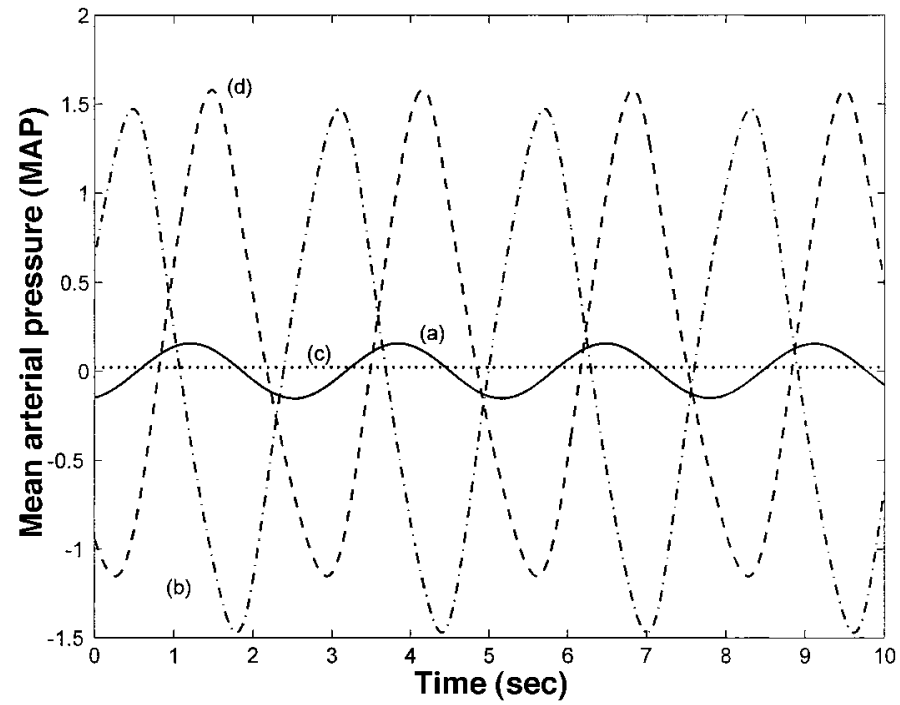

Fig. 6. Variations in MAP $(\mathrm{mmHg})$ around the mean level derived using a sigmoid nonlinearity in the forward path. The four cases, $a$, $b, c$, and $d$, are examples of changes in central nervous system parameters listed in Table 2 and indicate that under wide variations in the parameters of the sigmoidal curve (Fig. 5), the oscillation is stable.

Note that a stable limit cycle occurs where the $-[1 /$ $N(a)$ ] curve intersects the $G H(j \omega)$ curve. The limit cycle is stable (i.e., operation tends toward this point) because increases in the oscillation amplitude, $a$, causes movement along the $-[1 / N(a)]$ toward a region of stability, resulting in a decrease in $a$ and movement back to point $P$. Note also that the point $Q$ is given by

$$
\begin{aligned}
Q & =\lim _{a \rightarrow \infty}\left(-\frac{1}{N(a)}\right) \\
& =-\frac{\pi}{4 r^{*}}
\end{aligned}
$$

Depending on the sigmoid parameters, the point $Q$ may lie to the left of point $P$ (see example responses in Fig. 6 ), in which case no limit cycle occurs. On the basis of the available analysis, movement of $Q$ to the left can be caused by an increase in $r^{*}$. Movement of $P$ to the right can be caused by a decrease in $k_{p}$ (CNS gain). The value of $\beta$ (curvature parameter) does not affect the rightmost limit of the $-[1 / N(a)]$ curve. Because different points on the $-[1 / N(a)]$ line correspond to different values of $a$, the specific intersection point of the $-[1 /$

Table 2. CNS parameter variations corresponding to Fig. 6

\begin{tabular}{cccccc}
\hline \hline & CNS Gain & & & & \\
Case & $\left(k_{p}\right), \frac{\mu \mathrm{V}}{\mathrm{mmHg}}$ & $\begin{array}{c}\text { Range } \\
r^{*}, \mu \mathrm{V}\end{array}$ & $\begin{array}{c}\text { Curvature } \\
\beta\end{array}$ & $\begin{array}{c}\text { Horiz. Offset } \\
x^{*}, \mathrm{mmHg}\end{array}$ & $\begin{array}{c}\text { Vert. Offset } \\
y^{*}, \mu \mathrm{V}\end{array}$ \\
\hline (a) & 1.65 & 1 & 2 & 0 & 0 \\
(b) & 1.65 & 2 & 2 & 0 & 0 \\
(c) & 1.65 & 1 & 2 & 0.5 & 0 \\
(d) & 1.65 & 2 & 2 & 0.5 & 0 \\
\hline
\end{tabular}

CNS, central nervous system. 


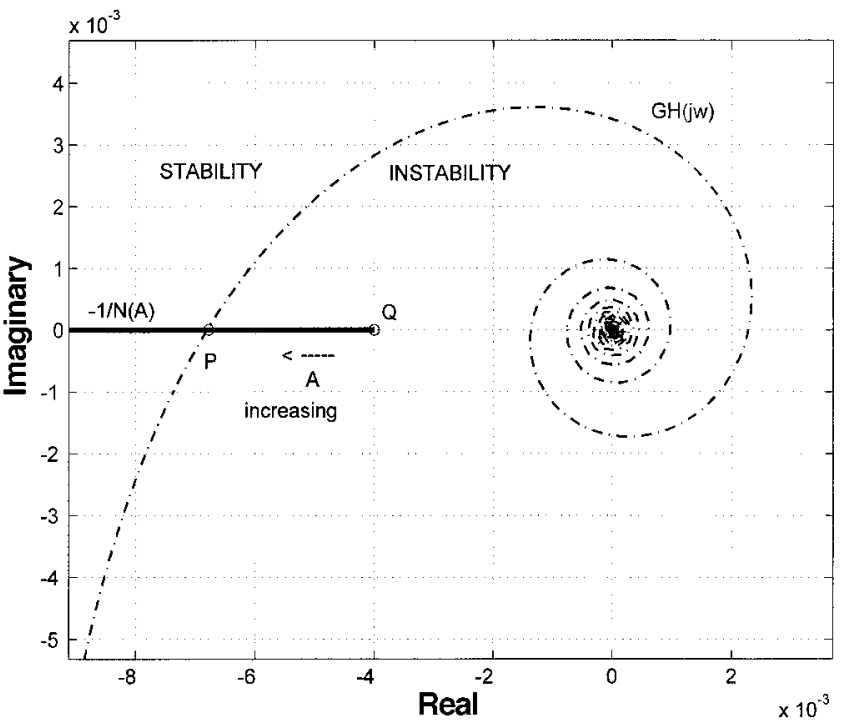

Fig. 7. Stability analysis for the nonlinear feedback loop indicating the limit cycle is stable because increases in the oscillation amplitude cause movement back toward a region of stability.

$N(a)]$ line and the $G H(j \omega)$ curve determines the oscillation amplitude.

An analytic approximation for the describing function, in terms of the parameters of the sigmoid, has been evaluated, which allows some insight into the change in oscillation amplitude for variations in the baroreflex curve. However, this analysis is excluded for brevity, but the interested reader is referred to Ref. 17.

\section{Interspecies Frequency Variability}

The oscillation frequency is determined solely by $\tau_{e}$, $\tau_{v}$, and $\tau_{a}$, because these determine the value for $\omega$ at the point $P$ in Fig. 7, given that $P$ lies to the left of $Q$, i.e., $G H(j \omega)$ and $-[1 / N(a)]$ intersect.

A

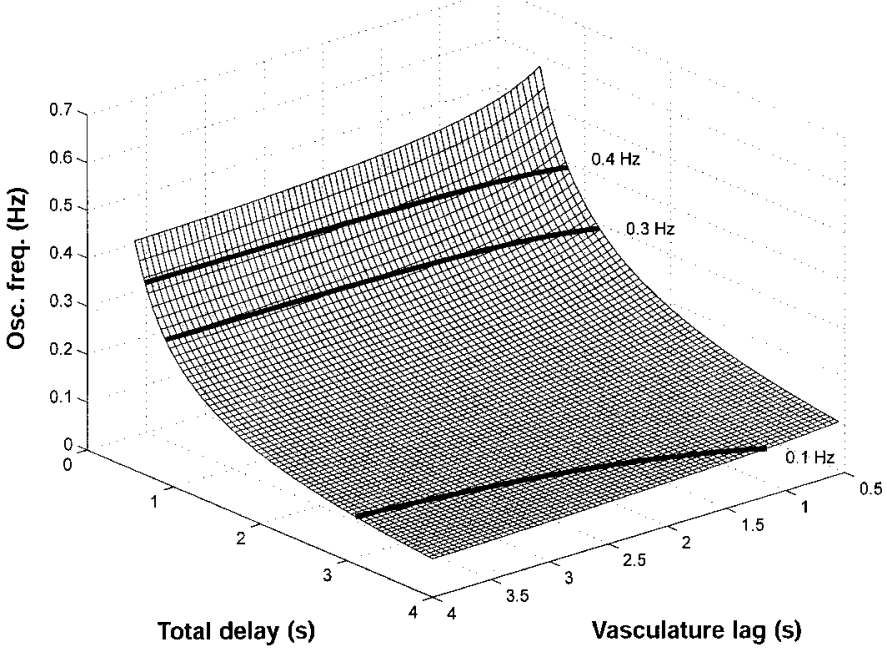

It is of particular interest to examine the variation in oscillation frequency with the model time constant and delays, because this relates particularly to interspecies variations. The oscillation frequency may be determined as the frequency (in rads/s), which causes the phase of $G H(j \omega)$ to equal $-\pi$ radians exactly. This occurs at a frequency $\omega_{0}$, where

$$
p\left(\omega_{o}\right)=-\pi+\omega_{o}\left(\tau_{e}+\tau_{a}\right)+\tan ^{-1}\left(\omega_{o} \tau_{v}\right)=0
$$

Unfortunately, $E q .8$ does not have an analytic solution, but can be solved by numerical optimization. A combined Gauss-Newton/quasi-Newton method is employed, which uses an analytic derivative calculation (easily calculated from Ref. 8) in the gradient minimization. This allows values of $\omega_{0}$ to be determined for different values of $\tau_{v}$ and $\tau_{e}+\tau_{a}$. Note that the efferent and afferent time delays are combined, because $\omega_{0}$ depends only on the total delay value. Figure 8 shows the variation in oscillation frequency (in $\mathrm{Hz}$ ) with variations in lag and delay terms. In particular, the 0.1-, $0.3-$, and $0.4-\mathrm{Hz}$ frequencies have been highlighted, showing the required relationship between $\tau_{v}$ and $\tau_{e}+$ $\tau_{a}$ for humans, rabbits, and rats.

Note that the oscillation frequency is much more sensitive to variations in delay than in time constant. Given that the smooth muscle characteristics are similar across species, the frequency variation across species is therefore explained by the difference in conduction times due to variation in size of species. Note also that this will (asymptotically) approach a limit, when the neurotransmission delay (relatively constant between species) dominates over the nerve conduction time.

A final comment relates to the use of a PD model for the baroreflex, instead of the pure gain term. The addition of such a term would add positive phase to the $G H(j \omega)$ plot in Fig. 7, causing it to rotate in an anticlockwise direction. The net result of this is that the

B

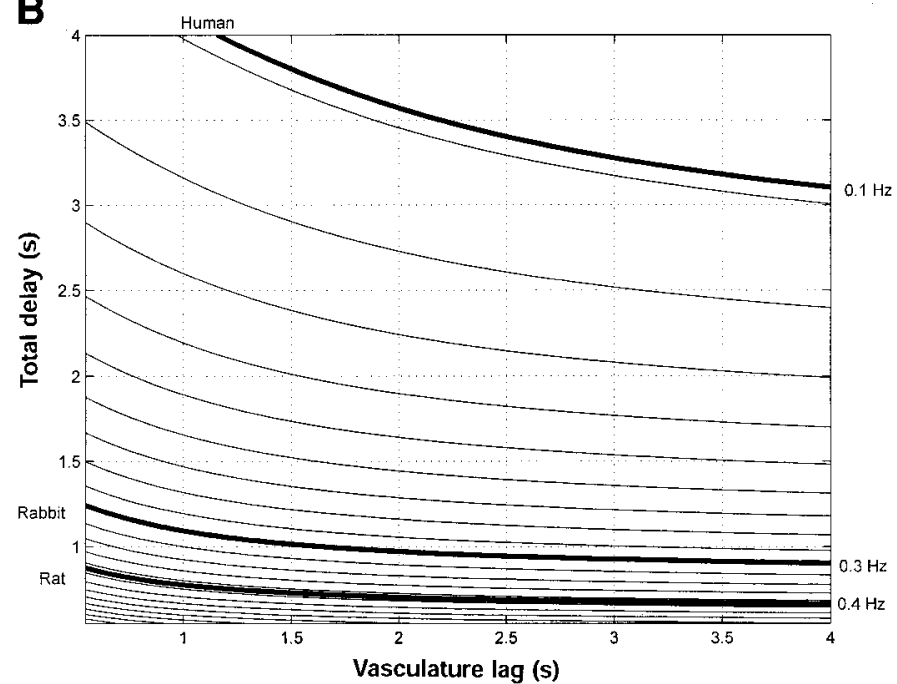

Fig. 8. $A$ and $B$ : variation in the oscillation frequency (Osc. Freq.) with changes in the total delay and vasculature lag. The solid lines drawn at $0.4,0.3$, and $0.1 \mathrm{~Hz}$ reflect the known oscillations in blood pressure for rats, rabbits, and humans, respectively (see text for explanation). 
intersection point of the $G H(j \omega)$ and - $[1 / N(a)]$ curves changes, with a slight increase in the oscillation frequency.

\section{Location of Nonlinearity Within Feedback Loop}

In this section, we explore the hypothesis that the vasculature does not contain a significant nonlinearity. If true, nonlinearity is only in CNS. If false, nonlinearity exists in both the vasculature and the CNS.

With the data recorded for a group of rabbits, a model may be constructed for the vasculature section, as in Fig. 1. The synthesis and comparison of linear and nonlinear models allow a conclusion to be made regarding the presence (or absence) of nonlinearity in the vasculature section. Note that the data employed in this modeling exercise are taken from animals under resting levels of MAP and SNA, and thus lie well within the limiting values of MAP and SNA that are known to introduce nonlinear effects $(4,29)$. The data also contain an oscillation at $\sim 0.3 \mathrm{~Hz}$.

To focus on the MAP/SNA system, models were constructed for MAP, using only SNA as an input. Although these models are not likely to explain the complete variation in MAP [which can be attributed to many factors, such as heart-rhythm, respiration, etc. $(18,33)]$, the focus here is on the comparative performance of linear and nonlinear models. Note that the data are collected from baroreceptor-innervated animals, for the following reasons: It is desired to measure the characteristics under normal feedback (oscillatory) conditions, and although in closed-loop mode, the relation between SNA and MAP contains both the feedforward effects of SNA on MAP as well as the feedback impact of MAP on SNA, the identification of the vasculature dynamics is nevertheless mathematically justified, because the CNS has a significant nonlinear component and prediction error methods are used for parameter identification (24).

Furthermore, it is important that a vasculature model be generated from data containing a low-frequency oscillation, so that any possible contributing nonlinear effects can be identified. Over the five animals considered, one rabbit had a strong low-frequency oscillation, two had less significant low-frequency oscillations, and the remaining two had no perceptible low-frequency component. Given the desired experimental conditions listed above, the rabbit with the significant oscillation was selected for modeling.

The original data were recorded at a sampling frequency of $500 \mathrm{~Hz}$, but the data are then filtered and resampled at a frequency of $2 \mathrm{~Hz}$. Use of a sampling rate of $2 \mathrm{~Hz}$, with a seventh-order anti-aliasing filter cut-off of $1 \mathrm{~Hz}$, eliminated the variations in the MAP data due to the heart rhythm. The spectrum of the resulting MAP signal is shown in Fig. 9, where the oscillation at $\sim 0.25 \mathrm{~Hz}$ is manifested as a resonant peak.

In the (resampled) data set from the selected animal, a total of 942 points is available. The first 500 points

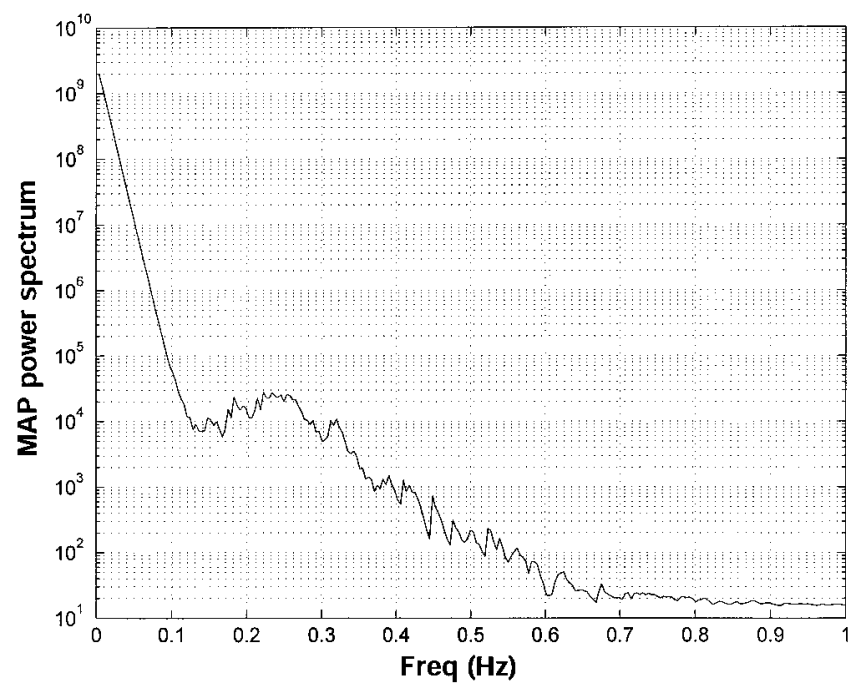

Fig. 9. Smoothed spectrum of resampled and filtered MAP against frequency (freq) from a single rabbit indicating an oscillation between 0.2 and $0.3 \mathrm{~Hz}$.

were used for training the model, with the next 442 points used for model validation.

The complete data set was detrended (the mean values of SNA and MAP were removed, respectively), resulting in a variational model. Although such a data transformation is not required in a nonlinear model, linear modeling requires the removal of the dc (zero frequency) component to avoid biased parameter estimates. To facilitate a meaningful comparison, the transformation was applied to both model types.

A linear model for the vasculature section. A linear discrete-time AutoRegressive with an eXogenous input (ARX) model of the form

$$
\frac{\operatorname{MAP}(z)}{\operatorname{SNA}(z)}=G(z)=\frac{b_{0}+b_{1} z^{-1}+\cdots+b_{m} z^{-m}}{1+a_{1} z^{-1}+\cdots+a_{n} z^{-n}} z^{-d}
$$

Initially, the values for $n, m$, and $d$ must be determined. This was performed using a loss function analysis, which compares the performance of different model structures. Figure 10 shows the loss function plotted for variations in $n, m$, and $d$, respectively. The chosen order and delay values, based on simultaneous optimization, are

$$
n=3, \quad m=2, \quad d=2
$$

Note that the choice of $d=2$ gives two-steps delay with a sampling period of $0.5 \mathrm{~s}$, resulting in a total delay of $1 \mathrm{~s}$, which accords well with measurements made via other routes (29). Although this may appear to imply that SNA would not affect MAP within this time period, the use of $d=2$ for the identification of the vasculature component is justified mathematically.

The optimal model (in a least-squares sense) with the desired structure is shown in Tables 3 and 4 . Note that the small SDs relative to the parameter values show the significance of the identified parameters. The model parameters were identified using a prediction error method (24). 
Table 3. Polynomial coefficients and SDs

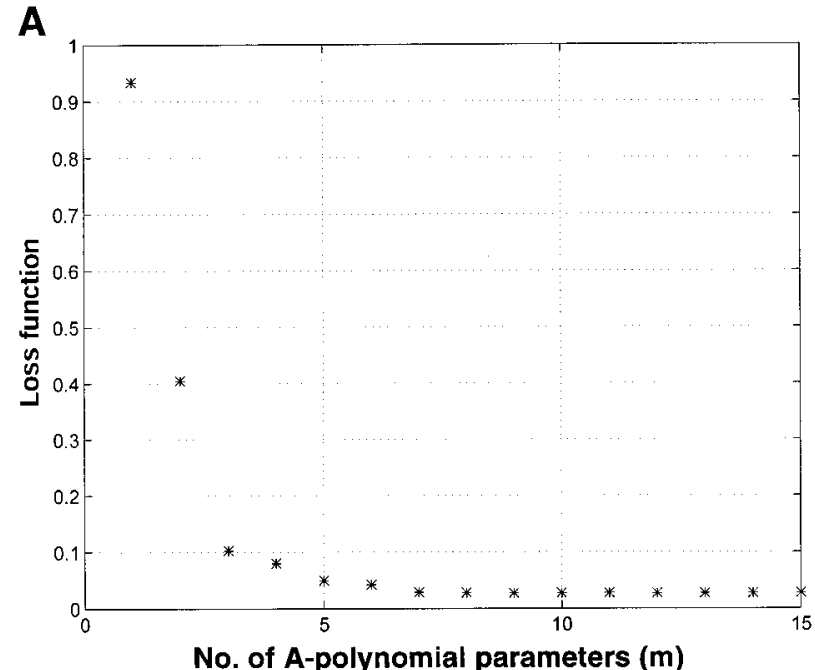

B
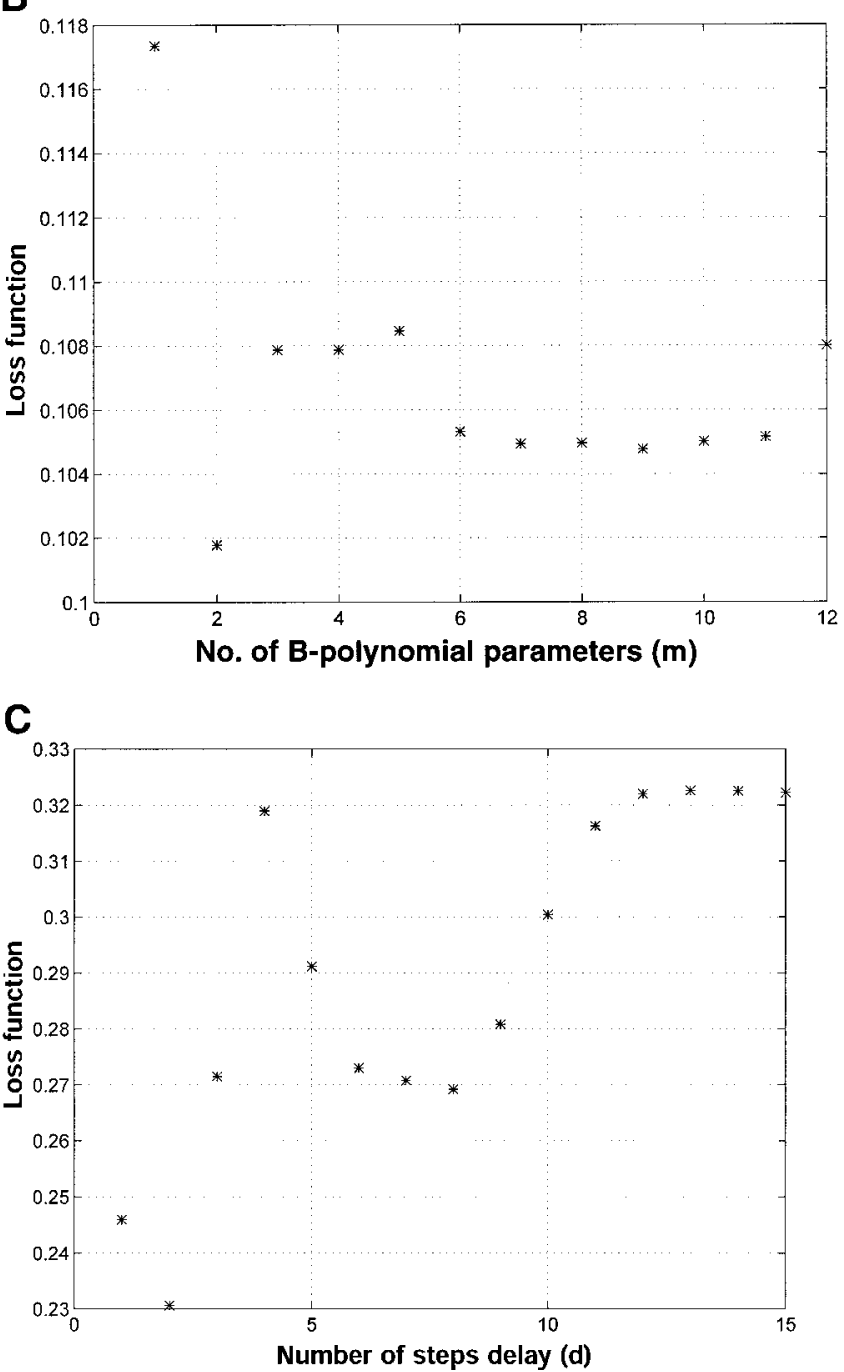

Fig. 10. Loss function variations for changes in model structure parameters $n(A), m(B)$, and $d(C)$.

\begin{tabular}{clrrr}
\hline \hline $\begin{array}{c}\text { Parameter } \\
\text { (Refer to } E \text { q. 9) }\end{array}$ & \multicolumn{1}{c}{$\mathrm{a}_{0}$} & \multicolumn{1}{c}{$\mathrm{a}_{1}$} & \multicolumn{1}{c}{$\mathrm{a}_{2}$} & \multicolumn{1}{c}{$\mathrm{a}_{3}$} \\
\hline Value & 1.0000 & -2.1633 & 1.9259 & -0.7126 \\
SD & 0 & 0.0286 & 0.0484 & 0.0284 \\
\hline
\end{tabular}

The performance of the linear ARX model on the training and validation data is shown in Fig. 11.

A nonlinear model for the vasculature section. A nonlinear model was constructed using an artificial neural network (ANN). ANN models are data based and can use exactly the same input structure as the previous linear model, providing a solid base for comparison. The corresponding nonlinear model, using the same model orders and delays as in Eqs. 9 and 10, is given as

$$
\mathrm{MAP}_{k}=v\left(\mathrm{MAP}_{k-1}, \mathrm{MAP}_{k-2}, \mathrm{SNA}_{k-2}, \mathrm{SNA}_{k-3}\right)
$$

where the nonlinear function, $v$, is synthesized using an ANN.

A fully connected three-layer network was employed, with tan-sig nonlinear basis functions. The significance of tan-sig nonlinear functions will be expanded on in Model comparisons. The output neuron is linear, but the two hidden layers contain nonlinear neurons, while the network was trained using backpropagation with momentum and an adaptive learning rate. The network is only trained for 600 epochs (or iterations of the full training data set) to avoid overtraining and thus provide good generalization.

The performance of the nonlinear ARX model on the training and validation data is shown in Fig. 12.

Model comparisons. Figures 11 and 12 show little difference between results for the linear and nonlinear models. This difference is quantified in terms of the mean squared error (MSE) for both sets of results in Table 5, both for the single-step and multi-step prediction errors. The MSE is defined as

$$
\mathrm{MSE}_{\mathrm{MAP}}=\frac{1}{N} \sum_{i=1}^{N}\left(\mathrm{MAP}_{a}-\mathrm{MAP}_{m}\right)^{2}
$$

where $\mathrm{MAP}_{a}$ is the actual MAP value; $\mathrm{MAP}_{m}$ is the modeled MAP value, and $N$ is the number of data points considered.

From examination of the comparative figures in Table 5 , it is clear that there is no significant improvement in the nonlinear model over the linear case, indicating that the data do not excite significant nonlinearity over the range of the data used for modeling and validation. To further investigate for possible nonlinearity in the vasculature, the steady-state gain characteristic of the ANN model is determined. This is

Table 4. Polynomial coefficients and SDs

\begin{tabular}{ccr}
\hline \hline Parameter & $\mathrm{b}_{0}$ & \multicolumn{1}{c}{$\mathrm{b}_{1}$} \\
\hline Value & 0.0927 & -0.0634 \\
SD & 0.0140 & 0.0143
\end{tabular}



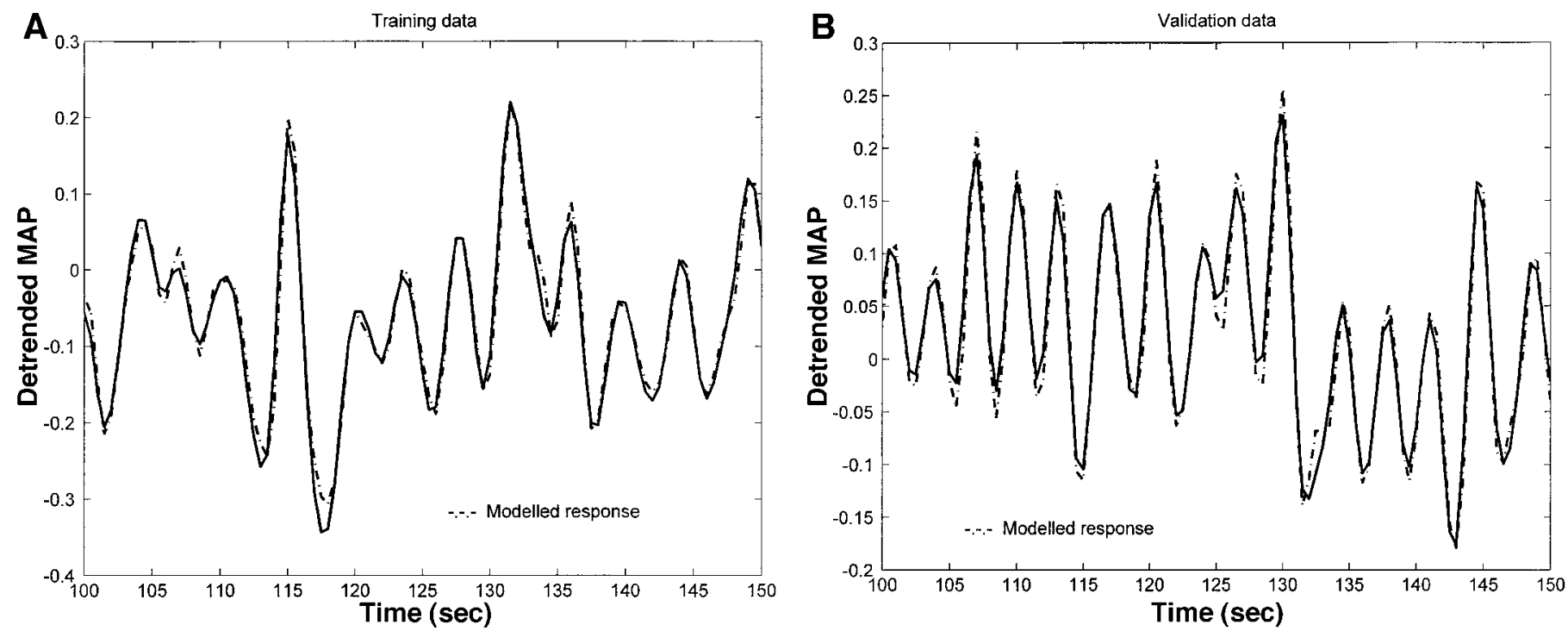

Fig. 11. Performance of the linear autoregressive with exogenous input (ARX) model for the training data $(A)$ and the validation data $(B)$. The solid line indicates the actual detrended MAP $(\mathrm{mmHg})$, dotted line the predicted MAP.

shown in Fig. 13. It is clear that this characteristic is only minimally nonlinear and, importantly, does not contain any inflexion points, which could give rise to sustained oscillations. However, it is in general agreement with experimental evidence, which indicates a decreasing vasculature gain with increasing SNA excitation.

\section{DISCUSSION}

The main finding of this study is that a nonlinear feedback model can account for the oscillation in blood pressure at $0.3 \mathrm{~Hz}$ in rabbits. Models constructed from conscious rabbits under resting conditions deduce that no nonlinearity is contained within the vasculature, but rather is contained within the CNS. The advantage of the nonlinear feedback model is that it is stable

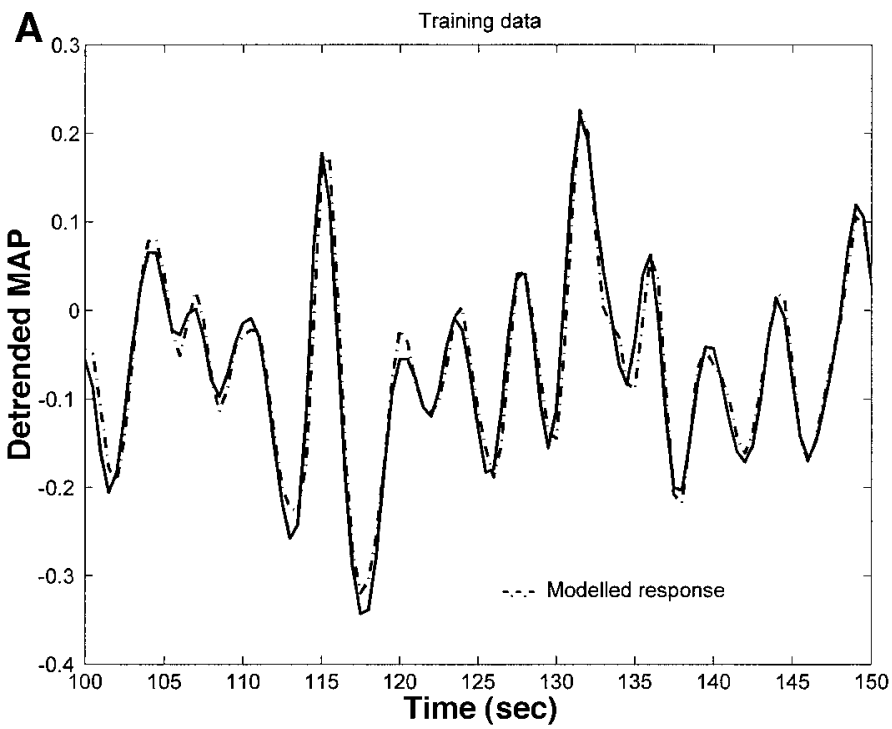

under a wide variety of situations where gain at various points along the feedback loop may be altered. Our model indicates that such changes in gain would not produce changes in the frequency of the oscillation but rather change its strength. Conversely, a linear model as previously proposed by Burgess et al. (8) would be unable to sustain an oscillation with changes in gain unless the loop had a system for continuous adaptation. In other words, the CNS would have to go to great lengths to sustain the oscillation. Thus, if the feedback loop giving rise to the oscillation is truely linear, and not nonlinear as we propose, then the oscillation is deliberate and not simply a by-product of various time delays in the circuit. The conclusion to draw from such a linear process is that the oscillation has a functional purpose and that the CNS works hard to maintain its existance.

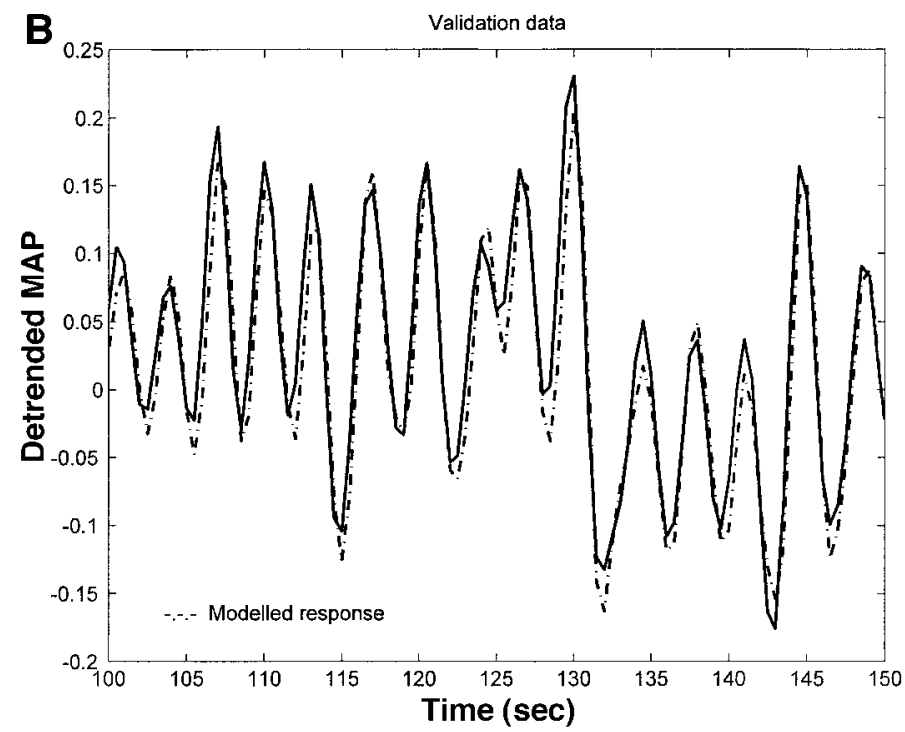

Fig. 12. Performance of the nonlinear ARX model for the training data $(A)$ and the validation data $(B)$. The solid line indicates the actual detrended MAP $(\mathrm{mmHg})$, dotted line the predicted MAP. 
Table 5. Comparative MSE figures

\begin{tabular}{lccc}
\hline \hline Model Type & $\begin{array}{c}\text { MSE } \\
\text { (Training) }\end{array}$ & $\begin{array}{c}\text { MSE } \\
\text { (Validation) }\end{array}$ & $\begin{array}{c}\text { MSE } \\
\text { (Multi-step) }\end{array}$ \\
\hline Linear & $3.6414 \times 10^{-4}$ & $3.4422 \times 10^{-4}$ & 0.0109 \\
Nonlinear & $3.3357 \times 10^{-4}$ & $3.2834 \times 10^{-4}$ & 0.0128 \\
\hline
\end{tabular}

MSE, mean squared error.

It is well established that the oscillation at $0.3 \mathrm{~Hz}$ in the rabbit is analogous to $0.4 \mathrm{~Hz}$ in the rat and $0.1 \mathrm{~Hz}$ in the human. Our nonlinear model is able to account for these species differences through changes in conduction time and indicates that changes in vasculature lag have little impact [generally considered relatively similar across species (40)]. The asymptotic behavior of oscillation frequency vs. species size is also relatively easily explained by observing (in Fig. 8) that oscillation frequency reduces asymptotically with decreasing conduction time. Recent research indicates that the oscillation is $\sim 0.4 \mathrm{~Hz}$ in mice (23). It is likely that this frequency is close to the maximal rate achievable, given that the conduction time in such species has approached its asymptotic limit.

There is now evidence from several animal models that sympathetic overactivity can initiate and/or subsequently maintain a blood pressure increase. In humans, essential hypertension is associated with elevated plasma norepinephrine levels, whereas muscle SNA is elevated in borderline hypertensives (16). With regard to blood pressure variability, when one considers general variability using a simple SD of blood pressure over $24 \mathrm{~h}$, the variability becomes progressively greater from normotensive to borderline, mild, and more severe essential hypertensive subjects (31). Understanding the origin and effect of this variability is likely to be of considerable clinical importance as previous studies have shown altered blood pressure and heart rate variability to be associated with increased risk of cardiovascular mortality $(15,32,34)$, raising the possibility of a diagnostic test using measurement of blood pressure variability.

Whereas the mechanisms responsible for overall blood pressure variability are not yet defined, there has been the suggestion that the amplitude of the 0.1- to $0.4-\mathrm{Hz}$ oscillation in blood pressure reflects either the mean level of sympathetic drive and/or changes in gain along the circuit $(6,26)$. In rabbits, stimuli that increase the mean level of renal SNA, such as hypoxia and hemorrhage, have been shown to increase the strength of $0.3-\mathrm{Hz}$ oscillations in SNA $(22,28)$. In the past, we proposed that the increase in the power of the oscillation was due to the increase in the mean SNA level. Analysis of the nonlinear model reveals that this is probably an association rather than a causal effect. Thus it is changes in baroreflex gain (via $k_{p}, r$, or $\beta$ ) that give rise to changes in the power of the oscillation with an increase in mean SNA levels having no effect on its strength. We previously showed that hypoxia increases the gain of the MAP-SNA baroreflex curve as well as the mean SNA level (27). The model predicts that a stimulus that changes the gain along the reflex loop will result in increases or decreases in the strength of the $0.3-\mathrm{Hz}$ oscillation. While this change in gain can most easily be detected by determining the MAP-SNA baroreflex relationship, our model indicates that an alteration in the gain in the vasculature response to sympathetic activity would also change the power of this oscillation.

In recent years, it has become popular to refer to the $0.1-\mathrm{Hz}$ oscillation in heart rate as a marker of sympathetic tone. In comparison with the power of the faster oscillation in heart rate associated with respiration, there have been numerous papers reporting changes in sympatho-vagal balance in such varied conditions as anesthesia (20), sleep (3), and the menstrual cycle (36). A mounting number of studies indicates that this hypothesis is flawed on several points, and it is more probable that the $0.1-\mathrm{Hz}$ oscillation in heart rate provides an index of baroreflex gain $(5,38,39)$. Our model supports this hypothesis and may explain why some stimuli such as coronary occlusion, which increases mean SNA levels, was associated with reductions in power at $0.1 \mathrm{~Hz}$ in heart rate (19). Studies in humans add further support to this hypothesis, where stimulation of carotid baroreceptors by neck suction at two frequencies $(0.1$ and $0.2 \mathrm{~Hz})$ induced a low-frequency oscillation in heart rate or blood pressure only if baroreflex sensitivity was normal, and that low baroreflex sensitivity was associated with reduced variability at this low frequency (38). Our nonlinear model provides the framework for predicting the changes in the power of the oscillation in blood pressure based on known changes in baroreflex gain. Though operation is normally in the "linear" portion of the sigmoid curve (35), the presence of an inflexion point has been demonstrated as sufficient to induce oscillations. Thus the oscillation amplitude can be well within the range of the sigmoid curve.

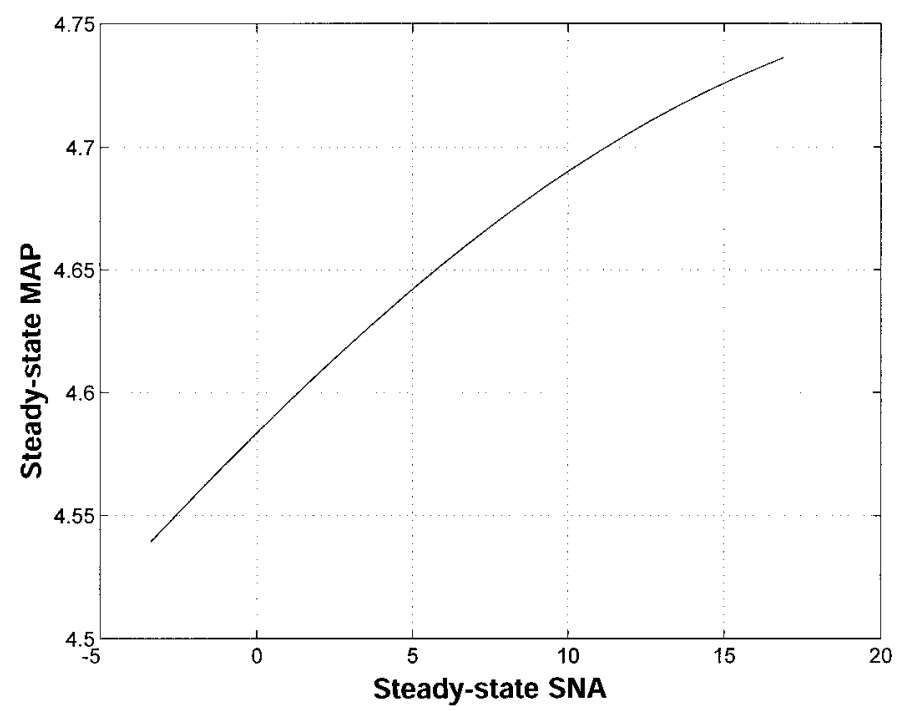

Fig. 13. DC gain characteristic of artificial neural network model showing MAP (steady-state MAP in $\mathrm{mmHg}$ ) against SNA (steadystate SNA in $\mu \mathrm{V}$ ). 


\section{Limitations}

Although the model is unlikely to be a completely accurate mimic (even structurally) of the CNS control over SNA, it is an important component part that provides a sound foundation for explaining the slow oscillation in blood pressure. However, the model as presented represents a uniform description of the sympathetic effects on total peripheral resistance and, as such, cannot represent differential changes in resistance through different organs. For example, hypoxia is well understood to produce a differential change in sympathetic activity to a number of organs, in particular, increases to the kidney with decreases to the skin (21). This allows blood pressure to remain constant in the face of differential increases in SNA, which can instigate localized oscillations in blood flow through organs such as the kidney (30). In contrast, our model results in a mean increase in blood pressure after a mean increase in SNA. Additionally, the model takes no account of blood pressure variations as a result of changes in cardiac output.

The extension of the model to include all of these components not only represents a considerable body of work, but also contains significant experimental difficulties in measurement of SNA to multiple organs to parameterize such a model. The purpose of this paper is to highlight the fundamental model structure that can explain low-frequency oscillations, while not accounting for all possible physiological scenarios. In essence, we conclude that a slow oscillation in blood pressure is best described by a nonlinear feedback model.

We are grateful for helpful discussions with Dr. Don Burgess, Dr. Mark Andrews, and Associate Professor Paul Austin.

This research was supported by the Mardsen Fund of New Zealand.

\section{REFERENCES}

1. Ando SI, Dajani HR, and Floras JS. Frequency domain characteristics of muscle sympathetic nerve activity in heart failure and healthy humans. Am J Physiol Regulatory Integrative Comp Physiol 273: R205-R212, 1997.

2. Atherton DP. Nonlinear Control Engineering. New York: Van Nostrand Reinhold, 1975.

3. Baharav A, Kotagal S, Gibbons V, Rubin BK, Pratt G, Karin J, and Akselrod S. Fluctuations in autonomic nervous activity during sleep displayed by power spectrum analysis of heart rate variability. Neurology 45: 1183-1187, 1995.

4. Bendle RD, Malpas SC, and Head GA. Role of endogenous angiotensin II on sympathetic reflexes in conscious rabbits. Am J Physiol Regulatory Integrative Comp Physiol 272: R1816-R1825, 1997.

5. Bernardi L, Leuzzi S, Radaelli A, Passino C, Johnston JA, and Sleight $\mathbf{P}$. Low-frequency spontaneous fluctuations of R-R interval and blood pressure in conscious humans: a baroreceptor or central phenomenon? Clin Sci 87: 649-654, 1994.

6. Bertram D, Barres C, Cuisinaud G, and Julien C. The arterial baroreceptor reflex of the rat exhibits positive feedback properties at the frequency of mayer waves. J Physiol (Lond) 513: 251-261, 1998.

7. Brown DR, Brown LV, Patwardhan A, and Randall DC. Sympathetic activity and blood pressure are tightly coupled at $0.4 \mathrm{~Hz}$ in conscious rats. Am J Physiol Regulatory Integrative Comp Physiol 267: R1378-R1384, 1994.
8. Burgess DE, Hundley JD, Li S-G, Randall DC, and Brown DR. First-order differential-delay equation for the baroreflex predicts the $0.4-\mathrm{Hz}$ blood pressure rhythm in rats. Am J Physiol Regulatory Integrative Comp Physiol 273: R1878-R1884, 1997.

9. Burgess DE, Zimmerman TA, Wise MT, Li S, Randall DC, and Brown DR. Low-frequency renal sympathetic nerve activity, arterial BP, stationary " $1 / f$ noise," and the baroreflex. Am $J$ Physiol Regulatory Integrative Comp Physiol 277: R894-R903, 1999.

10. Castellano M, Rizzoni D, Beschi M, Muiesan ML, Porteri E, Bettoni G, Salvetti M, Cinelli A, Zulli R, and Agabitirosei E. Relationship between sympathetic nervous system activity, baroreflex and cardiovascular effects after acute nitric oxide synthesis inhibition in humans. J Hypertens 13: 1153-1161, 1995.

11. Chapleau MW, Cunningham JT, Sullivan MJ, Wachtel RE, and Abboud FM. Structural versus functional modulation of the arterial baroreflex. Hypertension 26: 341-347, 1995.

12. Coughanowr DR and Koppel LB. Process Systems Analysis and Control. Kogakusha: McGraw-Hill, 1965.

13. DeBoer R, Karemaker J, and Strackee J. Hemodynamic fluctuations and baroreflex sensitivity in humans: a beat-to-beat model. Am J Physiol Heart Circ Physiol 253: 680-689, 1987.

14. Dorf RC and Bishop RH. Modern Control Systems (7th ed.). Reading, MA: Addison-Wesley, 1997.

15. Farrell TG, Bashir Y, Cripps T, Malik M, Plooniecki J, Bennett ED, Ward DE, and Camm AJ. Risk stratification for arrhythmic events in postinfarction patients based on heart rate variability, ambulatory electrocardiographic variables and the signal-averaged electrocardiogram. J Am Coll Cardiol 18: 687697, 1991.

16. Goldstein DS. Plasma norepinephrine during stress in essential hypertension. Hypertension 3: 551-556, 1981.

17. Holohan AM. On calculating describing functions. In: Proceedings of Irish Signals and Systems Conference Dublin, June 2000.

18. Holstein-Rathlou NH and Marsh DJ. Renal blood flow regulation and arterial pressure fluctuations: a case study in nonlinear dynamics. Physiol Rev 74: 637-681, 1994.

19. Houle MS and Billman GE. Low-frequency component of the heart rate variability spectrum: a poor marker of sympathetic activity. Am J Physiol Heart Circ Physiol 45: H215-H223, 1999.

20. Introna R, Yodlowski E, Pruett J, Montano N, Porta A, and Crumrine R. Sympathovagal effects of spinal anesthesia assessed by heart rate variability analysis. Anesth Analg 80: 315-321, 1995.

21. Iriki M, Riedel W, and Simon E. Patterns of differentiation in various sympathetic efferents induced by changes of blood gas composition and by central thermal stimulation in anaesthetised rabbits. Jpn J Physiol 22: 585-602, 1972.

22. Janssen BJA, Malpas SC, Burke SL, and Head GA. Frequency-dependent modulation of renal blood flow by renal nerve activity in conscious rabbits. Am J Physiol Regulatory Integrative Comp Physiol 273: R597-R608, 1997.

23. Janssen BJA, Leenders PJA, and Smits JFM. Short-term and long-term blood pressure and heart rate variability in the mouse. Am J Physiol Regulatory Integrative Comp Physiol 278: R215-R225, 2000

24. Ljung L. System Identification: Theory for the User (2nd ed.). Englewood Cliffs, NJ: Prentice-Hall, 1999.

25. Madwed J, Albrecht P, Mark R, and Cohen R. Low-frequency oscillations in arterial pressure and heart rate: a simple computer model. Am J Physiol Heart Circ Physiol 256: H1573H1579, 1989.

26. Malliani A, Pagani M, Lombardi F, and Cerutti S. Cardiovascular neural regulation explored in the frequency domain. Circ Res 84: 482-492, 1991.

27. Malpas SC, Bendle RD, Head GA, and Ricketts JH. Frequency and amplitude of sympathetic discharges by baroreflexes during hypoxia in conscious rabbits. Am J Physiol Heart Circ Physiol 271: H2563-H2574, 1996.

28. Malpas SC, Evans RG, Head GA, and Lukoshkova EV. Contribution of renal nerves to renal blood flow variability during hemorrhage. Am $J$ Physiol Regulatory Integrative Comp Physiol 274: R1283-R1294, 1998. 
29. Malpas SC, Hore TA, Navakatykyan M, Lukoshkova EV, Nguang SK, and Austin P. Resonance in the renal vasculature evoked by activation of the sympathetic nerves. Am J Physiol Regulatory Integrative Comp Physiol 276: R1311-R1319, 1999.

30. Malpas SC and Burgess DE. Renal sympathetic nerve activity as the primary mediator of slow oscillations in blood pressure during haemorrhage. Am J Physiol Heart Circ Physiol 279: H1299-H1306, 2000.

31. Mancia G, Ferrari A, Gregorini L, Parati G, Pomidossi G, Bertinieri G, Grassi G, di Rienzo M, Pedotti A, and Zanchetti A. Blood pressure and heart rate variabilities in normotensive and hypertensive human beings. Circ Res 53: 96-104, 1983.

32. Mancia G, Giannattasio C, Turrini D, Grassi G, and Omboni S. Structural cardiovascular alterations and blood pressure variability in human hypertension. J Hypertension 13: S7-S14, 1995.

33. O'Leary DS and Woodbury DJ. Role of cardiac output in mediating arterial blood pressure oscillations. Am J Physiol Regulatory Integrative Comp Physiol 271: R641-R646, 1996.

34. Parati G, Ravogli A, Frattola A, Groppelli A, Ulian L, Santucciu C, and Mancia G. Blood pressure variability: clinical implications and effects of antihypertensive treatment. J Hypertens 12: S35-S40, 1994.

35. Ricketts JH and Head GA. A five-parameter logistic equation for investigating asymmetry of curvature in baroreflex studies.
Am J Physiol Regulatory Integrative Comp Physiol 277: R441R454, 1999

36. Sato N, Miyake S, Akatsu JI, and Kumashiro M. Power spectral analysis of heart rate variability in healthy young women during the normal menstrual cycle. Psychosom Med 57: 331-335, 1995.

37. Sato T, Kawada T, Inagaki M, Shishido T, Takaki H, Sugimachi M, and Sunagawa K. New analytic framework for understanding sympathetic baroreflex control of arterial pressure. Am J Physiol Heart Circ Physiol 45: H2251-H2261, 1999.

38. Sleight P, Larovere MT, Mortara A, Pinna G, Maestri R, Leuzzi S, Bianchini B, Tavazzi L, and Bernardi L. Physiology and pathophysiology of heart rate and blood pressure variability in humans: is power spectral analysis largely an index of baroreflex gain? Clin Sci 88: 103-109, 1995.

39. Stauss HM and Persson PB. Power spectral analysis of heart rate and blood pressure: markers for autonomic balance or indicators of baroreflex control? Clin Sci 88: 1-2, 1995.

40. Stauss HM, Stegmann J, Persson PB, and Hbler H-J. Frequency response characteristics of sympathetic transmission to skin vascular smooth muscles in rats. Am J Physiol Regulatory Integrative Comp Physiol 277: R591-R600, 1999.

41. Wessling KH and Settels JJ. Baromodulation explains short term blood-pressure variability. In: Psychophysiology of Cardiovascular Control, edited by Orlebeke JF, Mulder J, and VanDoornen LJP. New York: Plenum, 1985, p. 69-97.

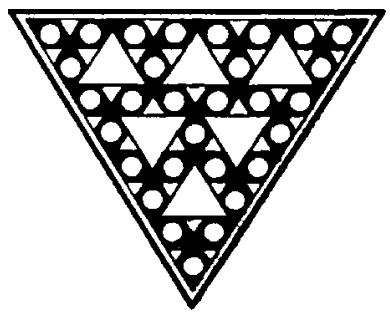

